\title{
Extension block and direct pinning methods for mallet fracture: A comparative study
}

\author{
Hyun Ho Han ${ }^{1}$, Hyun Jun $\mathrm{Cho}^{2}$, Seong Yeon Kim², Deuk Young $\mathrm{Oh}^{2}$ \\ ${ }^{I}$ Department of Plastic Surgery, Asan Medical Center, Ulsan University College of Medicine, Seoul; ${ }^{2}$ Department of Plastic \& Reconstructive \\ Surgery, College of Medicine, The Catholic University of Korea, Seoul, Korea
}

Background Mallet fracture can easily occur during sports activities or in daily life; however, the principles and methods of treatment for such fractures remain debated. Among the surgical treatments, various methods of closed reduction have been proposed. We treated patients with the extension block method (EBM) and the direct pinning method (DPM), and then compared the results. We assessed differences in range of motion and measurements of finger movement after surgery.

Methods A total of 41 patients who underwent surgery from August 2013 to September 2015 were evaluated retrospectively. Among them, 21 patients were treated with the EBM and 20 patients were treated with the DPM. We then compared extensor lag, range of motion, and outcomes according to Crawford's criteria between before surgery and at 6 to 8 months postoperatively.

Results The postoperative extensor lag improvement was $4.28^{\circ}$ and $10.73^{\circ}$, and the postoperative arc of motion was $55.76^{\circ}$ and $61.17^{\circ}$ in the EBM and DPM groups, respectively. The Crawford assessment showed no statistically significant difference between the groups, although the score in the DPM group was higher than that in the EBM group (3.5 vs. 3.1).

Conclusions As closed reduction methods for the treatment of mallet fracture, both the EBM and DPM showed good results. However, the DPM proved to be superior to the EBM in that it produced greater improvements in extensor lag and range of motion.

Keywords Fracture / Fingers / Tendon injuries / Closed fracture reduction
Correspondence: Deuk Young Oh Department of Plastic \&

Reconstructive Surgery, Seoul St. Mary's Hospital, College of Medicine, The Catholic University of Korea, 222

Banpo-daero, Seocho-gu, Seoul 06591, Korea

Tel: +82-2-2258-6143

Fax: +82-2-594-7230

E-mail: ohdeuk1234@hanmail.net

Received: 1 Sep 2017 - Revised: 15 Mar 2018 Accepted: 5 Apr 2018

pISSN: 2234-6163 • elSSN: 2234-6171 • https://doi.org/10.5999/aps.2017.01431 • Arch Plast Surg 2018;45:351-356

\section{INTRODUCTION}

Mallet finger is a common fingertip injury that is caused by trauma to the terminal insertion of the extensor digitorum tendon [1]. It commonly occurs during sports activities, although it can also occur during routine daily tasks [2]. Mallet fracture may be accompanied by soft tissue injury of the extensor digitorum tendon or avulsion fracture of the distal phalanx [3].
Currently, there are several points of debate about the principles and methods of mallet fracture treatment [1]. First, the choice between conservative care and surgical treatment is controversial [3-5]. If surgical treatment is chosen, it is unclear whether open reduction [6-10] or closed reduction [11-15] should be performed because neither method has shown specific benefits over the other. Therefore, choosing between open or closed reduction for the surgical approach can be difficult for 
physicians.

At Seoul St. Mary's Hospital, we primarily perform surgery in cases of mallet fracture, with closed reduction as the main method of choice. Closed reduction can be divided into two methods: the extension block method (EBM), which is the more commonly known method and was introduced by Ishiguro et al. in 1997 [11,12], and the Sasaki method, which involves pinning the fracture segment directly [14]. In addition, another method using an 18-gauge needle has been presented by some authors [15].

The EBM is undoubtedly one of the safest and best splinting methods, and it is widely used. However, iatrogenic avulsive injury of the extensor tendon can occur while extending the distal interphalangeal joint after pinning for reduction. Therefore, we hypothesized that extensor injury would occur more frequently during operations using the EBM than in operations using the direct pinning method (DPM), which is the modified version of the Sasaki method. To test this hypothesis, we compared the results of these two methods to evaluate their differences, if any, in terms of clinical outcome and prognosis, with a focus on extensor lag.

\section{METHODS}

This study was approved by the Institutional Review Board of our institution (KC16RISI0716). A total of 41 patients who were followed up for at least 4 months after surgery from August 2013 to September 2015 were enrolled based on a retrospective review of outpatient and inpatient department charts that contained statements of written informed consent from the patients. All operations were performed by a single surgeon, using the DPM from 2013 to September 2014 and the EBM from October 2014 to September 2015.

\section{Surgical indications}

Surgery was performed in cases with joint surface involvement of $>30 \%$ or volar subluxation of the distal phalanx, whereas in other relevant cases, splinting was done as conservative management. The Wehbe and Schneider (W-S) classification (Table 1) of mallet fractures is as follows: type I, no volar subluxation; type II, volar subluxation of the distal phalangeal joint; and type III, growth plate fracture or epiphyseal fractures of the distal phalanx. Each type is further divided into three subtypes: A, the fracture fragment size is less than one-third of the articular surface; $B$, the fragment size is from one-third to two-thirds of the articular surface; and $\mathrm{C}$, the fragment size is more than two-thirds of the articular surface [3]. We performed surgery in cases of subtypes B and C of type I and for all subtypes of types II and III.

\section{Surgical technique \\ Extension block method}

Surgery was done using a method similar to the EBM of Ishiguro et al. $[11,12]$. Under digital block anesthesia, the distal and proximal interphalangeal joints were held in maximum flexion. Under fluoroscopic imaging, a 0.9-mm Kirschner wire (K-wire) was inserted percutaneously through the terminal extensor tendon, into the middle phalanx, 1 to $2 \mathrm{~mm}$ dorsal to the fracture fragment (Fig. 1A). The distal phalanx was then pulled distally and extended in order to reduce the fracture. After reducing the fragment into position, a second K-wire was inserted from the distal phalanx to the middle phalanx longitudinally, to immobilize the distal interphalangeal joint (Fig. 1B).

\section{Direct pinning method}

We modified our previously reported surgical method [15]. Under digital block anesthesia and fluoroscopic imaging, the distal interphalangeal joints were held in extension. An 18-gauge needle was then inserted percutaneously from the dorsal side. Reduction was then performed by pushing the fracture fragment in place (Fig. 2A). In the reduced state, the fracture fragment and distal phalanx were fixed using a K-wire from the dorsal to the volar side (Fig. 2B). We used small K-wires (diameter, $0.7 \mathrm{~mm}$ ) for fixation. Even smaller K-wires (diameter, <0.7 $\mathrm{mm}$ ) were used when there was concern about fracture during fixation because of a bony fragment that was too small.

The second K-wire was inserted from the distal phalanx to the middle phalanx longitudinally to immobilize the distal interphalangeal joint.

\begin{tabular}{l} 
Table 1. Wehbe and Schneider classification \\
\hline Type I (no joint subluxation) \\
Subtype A: the fracture fragment size is less than one-third of the articular \\
surface. \\
Subtype B: the fracture fragment size is from one-third to two-thirds of the \\
articular surface. \\
Subtype C: the fracture fragment size is more than two-thirds of the articular \\
surface. \\
Type II (subluxation of the distal interphalangeal joint) \\
Subtype A: the fracture fragment size is less than one-third of the articular \\
surface. \\
Subtype B: the fracture fragment size is from one-third to two-thirds of the \\
articular surface. \\
Subtype C: the fracture fragment size is more than two-thirds of the articular \\
surface. \\
Type III (physis of the distal phalanx involved) \\
Subtype A: the fracture fragment size is less than one-third of the articular \\
surface. \\
Subtype B: the fracture fragment size is from one-third to two-thirds of the \\
articular surface. \\
Subtype C: the fracture fragment size is more than two-thirds of the articular \\
surface.
\end{tabular}




\section{Fig. 1. Extension block method}

(A) While holding the interphalangeal joints in the maximum flexed position, the surgeon inserts a 0.9-mm Kirschner wire percutaneously through the terminal part of the extensor tendon and into the middle phalanx, 1 to $2 \mathrm{~mm}$ dorsally to the fractured fragment. (B) The distal phalanx is then pulled distally and extended. When the fragment is reduced into position, a second Kirschner wire is inserted from the distal phalanx to the middle phalanx longitudinally.
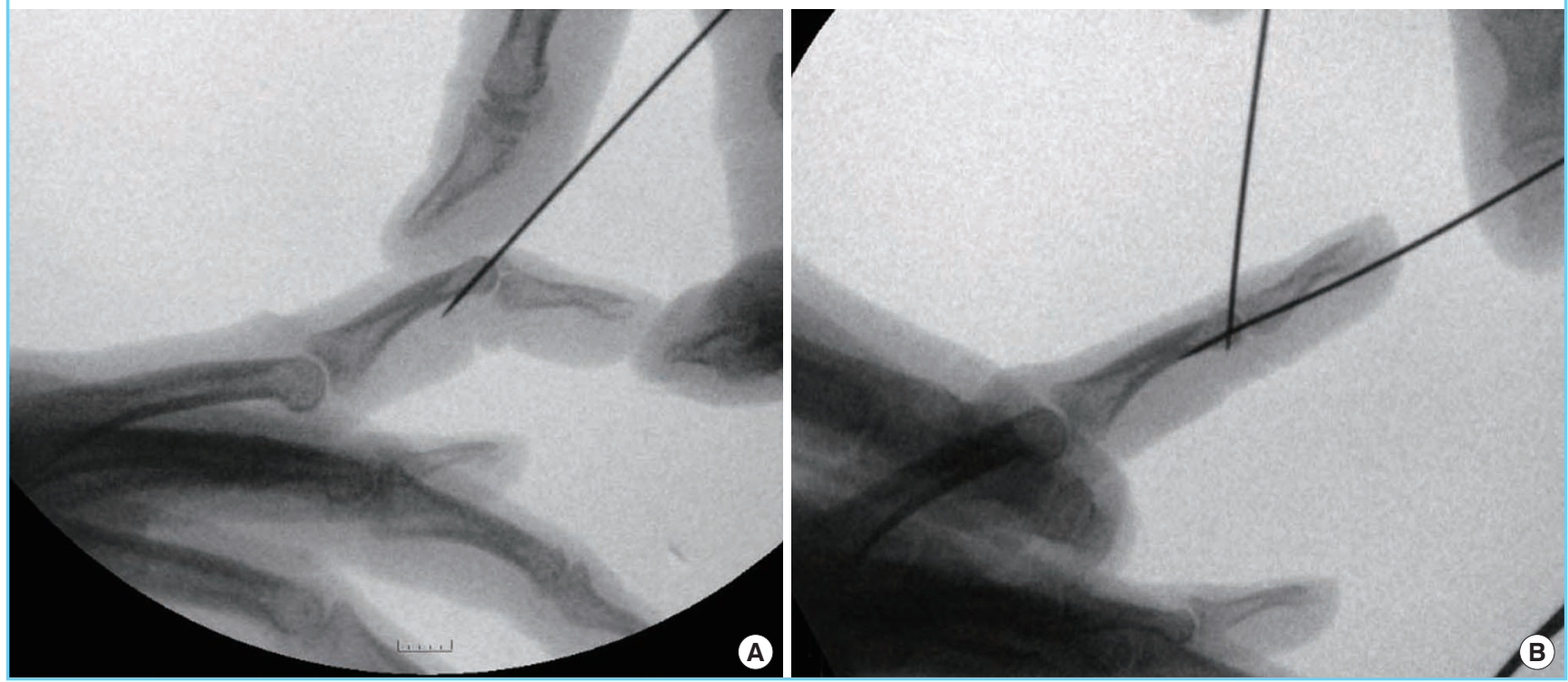

\section{Fig. 2. Direct pinning method}

(A) Reduction is done by pushing the 18-gauge needle that is inserted percutaneously from the dorsal side of the finger. (B) In the state of reduction, the fracture fragment and distal phalanx are directly fixed using a 0.9-mm Kirschner wire through the needle lumen from the dorsal to the volar side. A second Kirschner wire is inserted from the distal phalanx to the middle phalanx longitudinally to immobilize the distal interphalangeal joint.

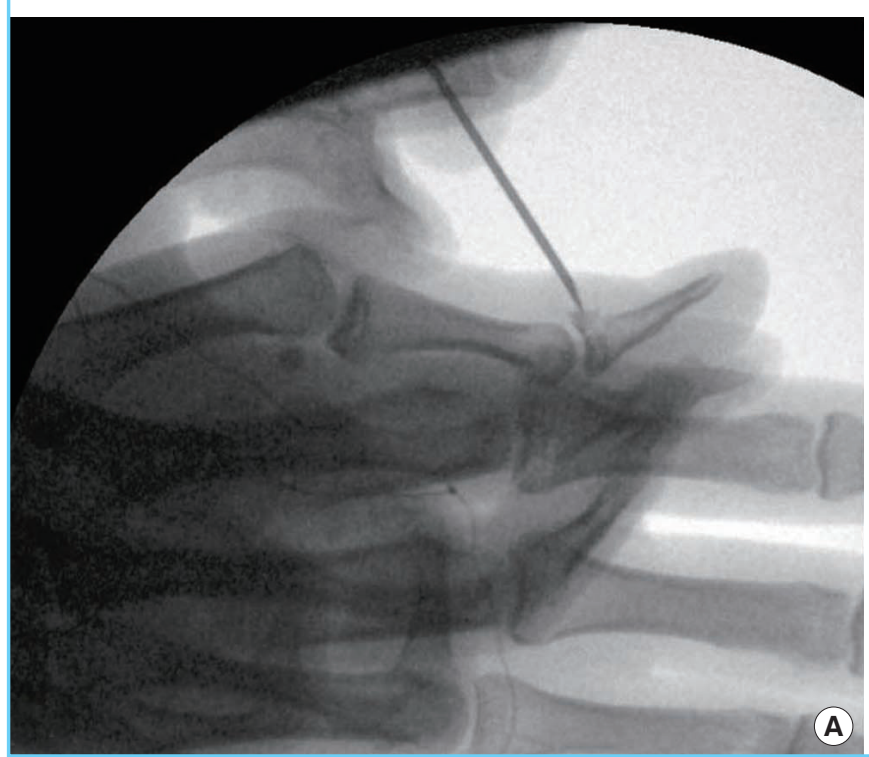

\section{Postoperative care and assessment}

All operations were performed based on information in the charts from the outpatient department. During the pin maintenance period, an aluminum finger splint was used by default for protection, and was maintained for 4 to 5 weeks. To prevent

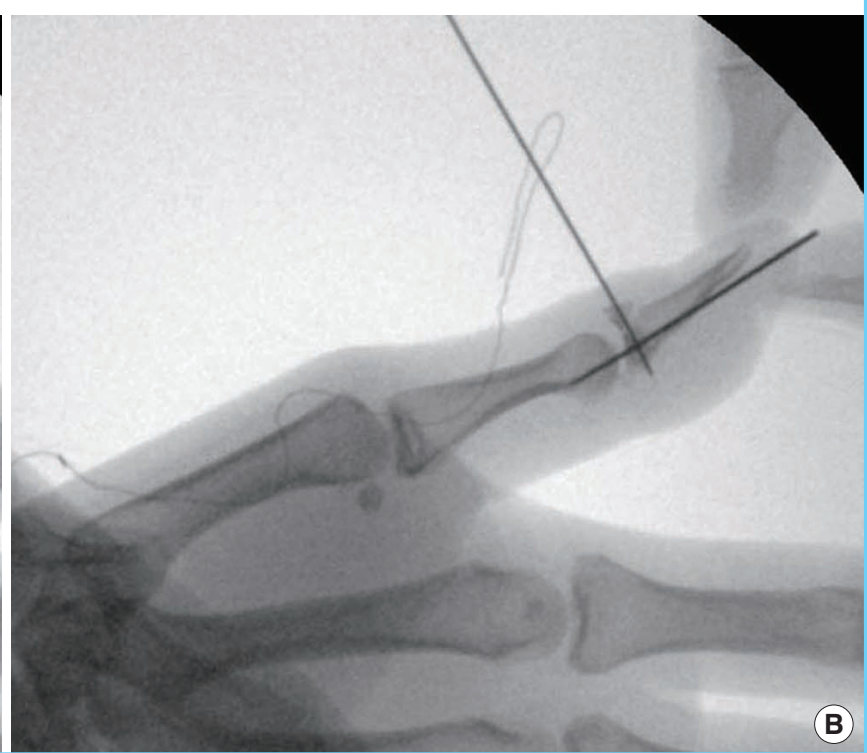

complications such as pin tract infection and pin loosening, we observed all patients closely. Upon pin removal, appropriate exercises of the distal interphalangeal joint were initiated. Extensor lag improvement, range of the arc of motion, and outcomes according to Crawford's criteria (Table 2) [16] were analyzed at 6 
to 8 months postoperatively. The grades based on Crawford's criteria were as follows: excellent for 4 points, good for 3 points, fair for 2 points, and poor for 1 point.

\section{Statistical analysis}

The postoperative changes in extensor lag were compared using the paired t-test, and the independent t-test was used to analyze the postoperative arc of motion and grades based on Crawford's criteria. A P-value of $\leq 0.05$ was considered to indicate statistical significance.

\section{RESULTS}

The patients' demographics are presented in Table 3. A total of 41 patients (29 males and 12 females) were included in this study, with a mean age of 36 years (range, 15-62 years). The finger injuries included one case of thumb injury, 11 cases of long finger injury, 21 cases of ring finger injury, and eight cases of small finger injury. According to the W-S classification, 23 cases

\begin{tabular}{|lcll|}
\hline \multicolumn{3}{|l|}{ Table 2. Crawford's criteria } & \\
\hline Classification & $\begin{array}{c}\text { Loss of } \\
\text { extension }\end{array}$ & Flexion & Pain \\
\hline Excellent & None & Full & None \\
Good & $0^{\circ}-10^{\circ}$ & Full & None \\
Fair & $10^{\circ}-25^{\circ}$ & Any loss of flexion & None \\
Poor & $>25^{\circ}$ & Any loss of flexion & Persistent pain \\
\hline
\end{tabular}

\begin{tabular}{|c|c|c|c|}
\hline & EBM & DPM & Total \\
\hline Total no. of patients & 21 & 20 & 41 \\
\hline \multicolumn{4}{|l|}{ Sex } \\
\hline Male & 16 & 13 & 29 \\
\hline Female & 5 & 7 & 12 \\
\hline Average age (yr) & 35.9 & 35.1 & $36(15-62)$ \\
\hline \multicolumn{4}{|l|}{ Involved digit } \\
\hline Thumb & 0 & 1 & 1 \\
\hline Index & 0 & 0 & 0 \\
\hline Long & 5 & 6 & 11 \\
\hline Ring & 11 & 10 & 21 \\
\hline Small & 5 & 3 & 8 \\
\hline \multicolumn{4}{|l|}{ W-S classification } \\
\hline \multicolumn{4}{|l|}{ I } \\
\hline B & 10 & 13 & 23 \\
\hline C & 0 & 0 & 0 \\
\hline \multicolumn{4}{|l|}{$\|$} \\
\hline A & 2 & 0 & 2 \\
\hline $\mathrm{B}$ & 8 & 6 & 14 \\
\hline C & 1 & 1 & 2 \\
\hline Average follow-up period (mo) & 6.7 & 6.3 & $6.5(5-11)$ \\
\hline
\end{tabular}

were type IB, two cases were IIA, 14 cases were IIB, and two cases were IIC. There were no cases of types IC or III. The mean follow-up period was 6.5 months (range, 5-11 months).

Twenty-one patients were treated with the EBM and 20 patients with the DPM (Table 4). The mean values of preoperative and postoperative extensor lag were $15.73^{\circ}$ and $8.31^{\circ}$, respectively, with $7.42^{\circ}$ of improvement. Extensor lag improved from $14.27^{\circ}$ to $9.99^{\circ}$ in the EBM group, whereas it improved from $17.27^{\circ}$ to $6.55^{\circ}$ in the DPM group. The improvement was $4.28^{\circ}$ and $10.73^{\circ}$ in the EBM and DPM groups, respectively. The difference between the groups was statistically significant $(\mathrm{P}=0.003)$.

The postoperative arc of motion was $55.76^{\circ}$ in the EBM group and $61.17^{\circ}$ in the DPM group. The difference was statistically significant $(\mathrm{P}=0.047)$. The Crawford assessment score was 3.1 and 3.5 in the EBM and DPM groups, respectively; however, this difference was not statistically significant $(\mathrm{P}=0.052)$. Only minor complications, such as mild erythema, occurred around the pin insertion site (seven patients), and no cases of major infection or inserted pin loosening were noted (Fig. 3).

\section{DISCUSSION}

Some physicians prefer conservative care and use splints to treat patients with mallet fracture $[5,17,18]$. If surgery is not performed, the splint should be maintained continually for 6 to 8 weeks, and the same cycle should be repeated if extensor lag occurs [1]. However, the conservative method leads to problems in long-term patient compliance; hence, we prefer surgical treatment.

Among the surgical methods, closed reduction was our method of choice. The EBM and DPM were performed uniformly, and possible differences between them in terms of surgical outcomes were evaluated.

In the EBM, direct fragment pinning is not done, as there is less risk of comminution. The fragment, however, can slip during reduction, and if the distal interphalangeal joint is extended

Table 4. Comparison of results between the EBM and the DPM

\begin{tabular}{|c|c|c|c|c|}
\hline & EBM & DPM & Total & P-value \\
\hline No. of patients & 21 & 20 & 41 & \\
\hline Preoperative extensor lag $\left(^{\circ}\right)$ & 14.27 & 17.27 & 15.73 & 0.095 \\
\hline Postoperative extensor lag $\left({ }^{\circ}\right)$ & 9.99 & 6.55 & 8.31 & 0.063 \\
\hline Degree of improvement $\left({ }^{(}\right)^{\mathrm{a})}$ & 4.28 & 10.73 & - & 0.003 \\
\hline Postoperative arc of motion $\left({ }^{\circ}\right)$ & 55.76 & 61.17 & - & 0.047 \\
\hline Crawford outcome score & 3.1 & 3.5 & - & 0.052 \\
\hline
\end{tabular}

EBM, extension block method; DPM, direct pinning method.

a)Preoperative extensor lag - postoperative extensor lag. 


\section{Fig. 3. Postoperative result}

(A, B) Postoperative results 3 months after the direct pinning method. Near full range of motion and no extension lag are shown. (C, D) Postoperative results 3 months after surgery with the extension block method. There was an extension lag of $10^{\circ}$ in the distal interphalangeal joint.
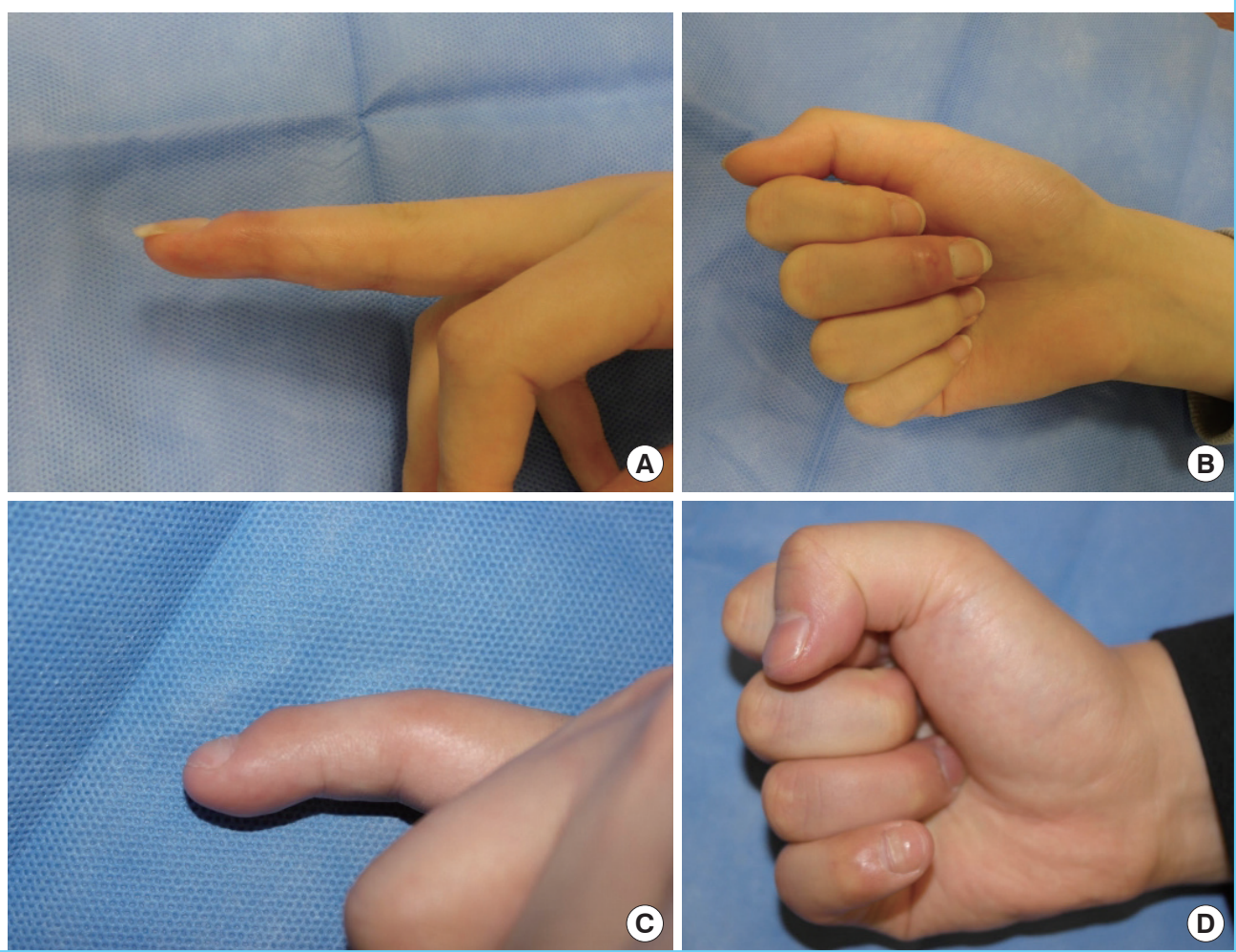

\section{Fig. 4. Possibility of an avulsive injury}

In the extension block method, there is no direct pinning to the fragment; hence, it is less likely to cause comminution of the fragment bone. However, it has a risk of fragment displacement during fixation and can cause extensor tendon injury if the distal phalangeal joint is extended in the pinning state.

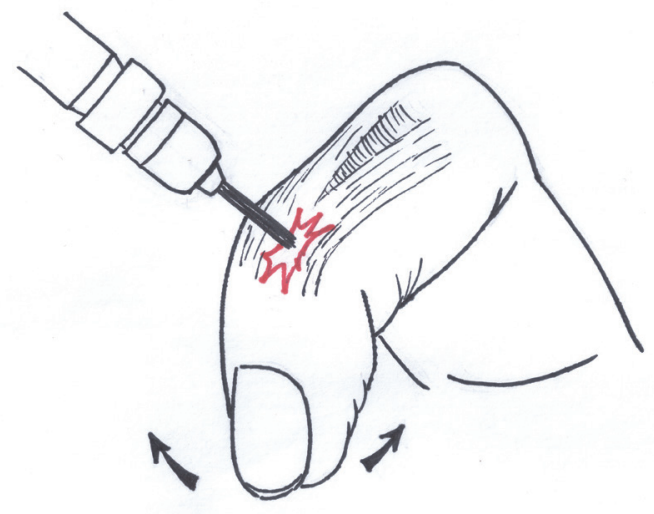

during pinning, the extensor tendon can tear, leading to bone avulsion (Fig. 4). Hence, increased extensor lag may occur if pinning and reduction fail. Repetitive drilling performed by less skilled and less experienced surgeons can cause further injury to the extensor insertion, leading to iatrogenic mallet deformities.

Although there is a risk of bone fragmentation in the DPM, reduction can easily be done using a pointed needle that directly that passes through the joint. However, this is somewhat less common, and extension and flexion of the distal interphalangeal joint do not occur; hence, the possibility of iatrogenic trauma to the extensor tendon is low. In another study, Yamanaka and Sasaki [14] used a similar surgical method in which the fractured distal phalangeal bony segments were fixed together using compression fixation pins, and this method resulted in a good range of motion within a shorter time than did other treatments.

As expected, our results showed less improvement in extensor lag in the EBM group than in the DPM group, and there was a difference in the range of motion postoperatively. When assessed using Crawford's criteria, both groups achieved good results (up to 3 points), clearly indicating that both methods are beneficial. A slightly higher score was obtained in the DPM group, which could have been due to the difference in the surgical technique; however, this difference was not statistically significant. As the follow-up period in this study was short, we consider it likely that the incidence of chronic deformity (i.e., swan neck deformity) will differ if the follow-up is $>1$ year [19]. Interesting differences are found in the morbidity of each finger. Previous reports have shown that mallet fracture does not occur extensively in the thumb and index finger [20]. It is also known that mallet finger has an overall incidence of around 90\% in the three ulnar digits [21]. In this report, ring finger injuries [20] were the most common, followed by long finger injuries 
[11] and small finger injuries [8].

The limitations of this study include its retrospective nature and the fact that the groups were divided by the timing of surgery, according to the surgeon's clinical judgment. The followup period was relatively short, so long-term complications could not be observed. Moreover, the DPM can cause additional fractures in the fracture site during insertion of the K-wire. Fortunately, there were no additional fractures in our cases; nevertheless, this possibility should be considered.

In conclusion, both the EBM and DPM are good surgical methods for closed reduction of mallet finger. However, the DPM proved to be superior in that it provided better improvement of extensor lag and range of motion.

\section{NOTES}

\section{Conflict of interest}

No potential conflict of interest relevant to this article was reported.

\section{Ethical approval}

The study was approved by the Institutional Review Board of Seoul St. Mary's Hospital (IRB No. KC16RISI071) and performed in accordance with the principles of the Declaration of Helsinki. Written informed consents were obtained.

\section{Patient consent}

The patients provided written informed consent for the publication and the use of their images.

\section{ORCID}

Hyun Ho Han https://orcid.org/0000-0001-7072-9882

Deuk Young Oh https://orcid.org/0000-0003-3499-1554

\section{REFERENCES}

1. Bloom JM, Khouri JS, Hammert WC. Current concepts in the evaluation and treatment of mallet finger injury. Plast Reconstr Surg 2013;132:560e-566e.

2. McCue FC 3rd, Meister K. Common sports hand injuries: an overview of aetiology, management and prevention. Sports Med 1993;15:281-9.

3. Wehbe MA, Schneider LH. Mallet fractures. J Bone Joint Surg Am 1984;66:658-69.

4. Warren RA, Kay NR, Ferguson DG. Mallet finger: comparison between operative and conservative management in those cases failing to be cured by splintage. J Hand Surg Br $1988 ; 13: 159-60$.
5. Richards SD, Kumar G, Booth S, et al. A model for the conservative management of mallet finger. J Hand Surg $\mathrm{Br}$ 2004;29:61-3.

6. Fritz D, Lutz M, Arora R, et al. Delayed single Kirschner wire compression technique for mallet fracture. J Hand Surg Br 2005;30:180-4.

7. Bischoff R, Buechler U, De Roche R, et al. Clinical results of tension band fixation of avulsion fractures of the hand. $J$ Hand Surg Am 1994;19:1019-26.

8. Zhang X, Meng H, Shao X, et al. Pull-out wire fixation for acute mallet finger fractures with $\mathrm{k}$-wire stabilization of the distal interphalangeal joint. J Hand Surg Am 2010;35:1864 9.

9. Bauze A, Bain GI. Internal suture for mallet finger fracture. J Hand Surg Br 1999;24:688-92.

10. Tung KY, Tsai MF, Chang SH, et al. Modified tenodesis method for treatment of mallet fractures. Ann Plast Surg 2012;69:622-6.

11. Ishiguro T, Itoh Y, Yabe Y, et al. Extension block with Kirschner wire for fracture dislocation of the distal interphalangeal joint. Tech Hand Up Extrem Surg 1997;1:95-102.

12. Pegoli L, Toh S, Arai K, et al. The Ishiguro extension block technique for the treatment of mallet finger fracture: indications and clinical results. J Hand Surg Br 2003;28:15-7.

13. Badia A, Riano F. A simple fixation method for unstable bony mallet finger. J Hand Surg Am 2004;29:1051-5.

14. Yamanaka K, Sasaki T. Treatment of mallet fractures using compression fixation pins. J Hand Surg Br 1999;24:358-60.

15. Rha EY, Lee MC, Lee JH, et al. Treatment of mallet fracture using a percutaneous fixation technique with an 18-gauge needle. Acta Orthop Belg 2015;81:296-302.

16. Crawford GP. The molded polythene splint for mallet finger deformities. J Hand Surg Am 1984;9:231-7.

17. Bendre AA, Hartigan BJ, Kalainov DM. Mallet finger. J Am Acad Orthop Surg 2005; 13:336-44.

18. Pike J, Mulpuri K, Metzger M, et al. Blinded, prospective, randomized clinical trial comparing volar, dorsal, and custom thermoplastic splinting in treatment of acute mallet finger. J Hand Surg Am 2010;35:580-8.

19. Tocco S, Boccolari P, Landi A, et al. Effectiveness of cast immobilization in comparison to the gold-standard self-removal orthotic intervention for closed mallet fingers: a randomized clinical trial. J Hand Ther 2013;26:191-200.

20. Salazar Botero S, Hidalgo Diaz JJ, Benaida A, et al. Review of acute trau matic closed mallet finger injuries in adults. Arch Plast Surg 2016;43:134-44.

21. Alla SR, Deal ND, Dempsey IJ. Current concepts: mallet finger. Hand (NY) 2014;9:138-44. 